\title{
Recent Results on Binary Cepheids
}

\author{
Nancy Remage Evans ${ }^{1,2}$ \\ ${ }^{1}$ Institute for Space and Terrestrial Science, York University, ${ }^{2}$ IUE Guest Observer
}

\begin{abstract}
IUE observations of hot companions of Cepheids have been used to determine the temperatures of the companions. For companions on the ZAMS, the temperatures can be used to determine the luminosity of the Cepheids from the inferred absolute magnitude of the companion. The accuracy of this method is comparable to the accuracy of calibration using Cepheids in clusters. An overtone pulsator ( $\mathrm{SU}$ Cas) has been identified by this technique. The luminosity of the double mode pulsator Y Car agrees with that from the PLC, confirming that is a normal Population I Cepheid. The variation of the width of the instability strip (as a function of luminosity) in the HR diagram is confirmed, and overtone and double mode pulsators are shown to be near the blue edge of the instability strip. Comparison between variables and nonvariables in the HR diagram (from IUE and cluster studies) shows very little overlap. The shape of the observed instability region may be determined both by the boundary between the variables and nonvariables and also the location of the tips of the blue loops of evolutionary tracks. Finally, eight Cepheids with hot companions which are evolved beyond the ZAMS have been studied. Half the systems are matched well by evolutionary tracks with little or no core convective overshoot near the main sequence. At least two and possibly four systems, however, cannot be matched by current isochrones.
\end{abstract}

\section{Cepheid Luminosity Determination}

Cepheids which have hot companions can be "temperature resolved", that is, each component can be studied in detail in the wavelength region in which it dominates. Low resolution IUE spectra of the hot companions provide a well-determined spectral type, from which the absolute magnitude can be inferred. From this the absolute magnitude of the Cepheid can be determined. The details of the process are provided in Evans 1991 and 1992a. Previous discussions of IUE spectra of Cepheid companions are given by Böhm-Vitense 1985 and Böhm-Vitense and Proffitt 1985. Figure 1 shows the comparison of the results from IUE and the cluster Cepheids from Feast and Walker 1987. The IUE results (7 stars) have an rms deviation from the Feast and Walker period-luminosity-color (PLC) relation of $0.37 \mathrm{mag}$ (for one observation), or a smaller deviation (0.33 mag) if the IUE results are used to determine the zero-point of the relation. Although the IUE results indicate a slightly fainter PLC than Feast and Walker, this result is preliminary because additional stars can be added to the IUE results and the analysis will be redone when the IUE data has been reprocessed. 
Furthermore, we (Evans, Pitts, and Imhoff) have obtained spectra of comparable Pleiades stars, so that the calibration of the companions can be tied directly to them.

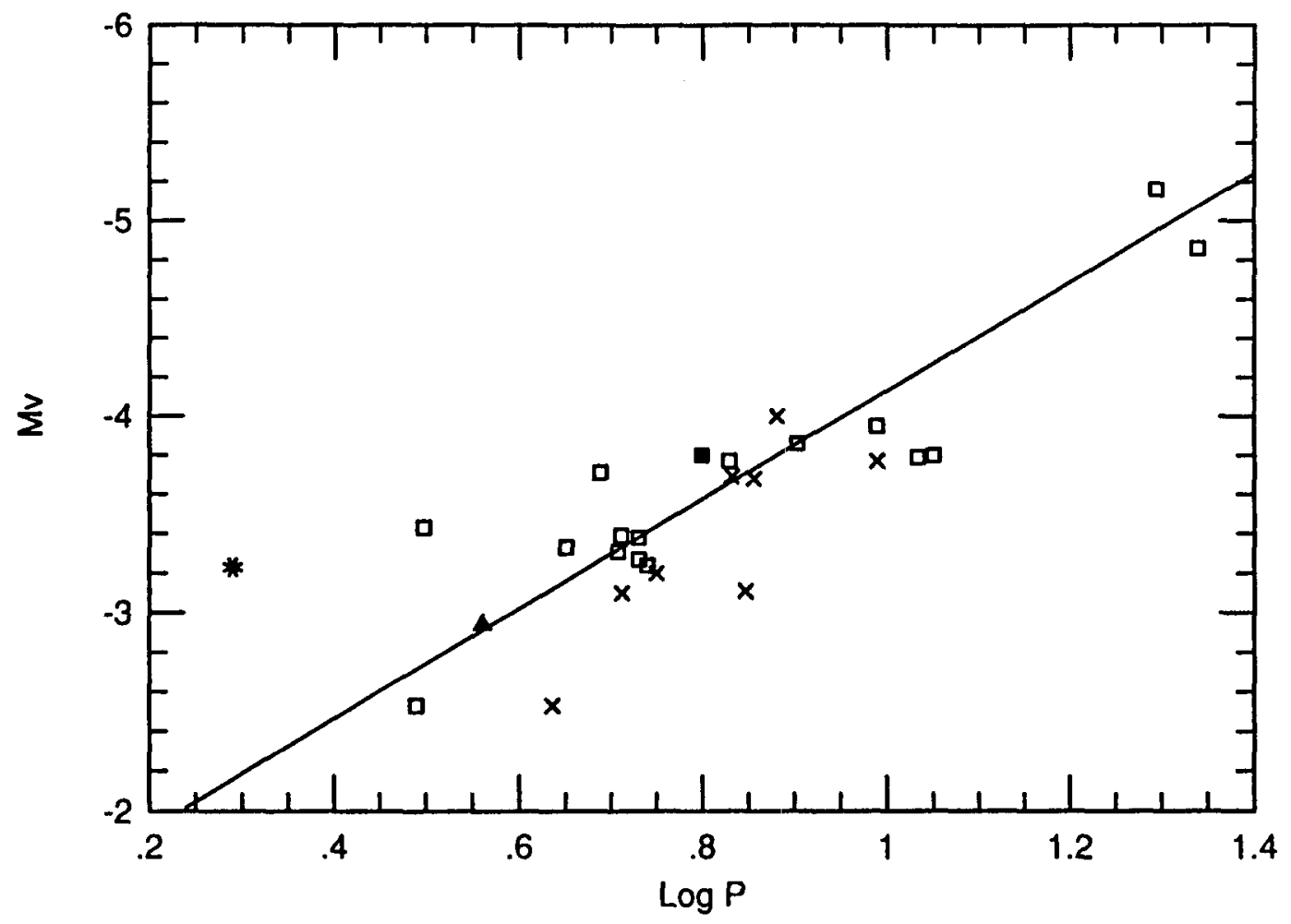

Figure 1 The period luminosity relation. Open and filled squares are the data from Feast and Walker for cluster Cepheids; the filled square is V367 Sct; other data are IUE determinations; the filled triangle is the double mode pulsator Y Car; the asterisk is SU Cas. The line is the period-luminosity relation from Feast and Walker.

In addition to the fundamental mode pulsators, we have observed a Cepheid we identify as an overtone pulsator (SU Cas, Evans 1991) and a double mode pulsator (Y Car, Evans 1992b). SU Cas is more luminous than the PLC relation would predict for a fundamental mode pulsator, and hence we conclude that it is pulsating in an overtone mode. This is independent evidence that this short period "s-Cepheid" (identified from the Fourier coefficients by Antonello, Poretti, and Reduzzi 1990) is actually an overtone pulsator. The luminosity of the double mode Cepheid Y Car is in good agreement with that predicted from the fundamental mode and the PLC, confirming that its properties are comparable to single mode pulsators.

An HR diagram can be plotted using the Cepheids with independent luminosities from the IUE determinations and the Feast and Walker cluster stars. We confirm the 
result of Fernie 1990 that the instability strip is narrower at lower luminosities than at high luminosities. In addition, the two double-mode pulsators Y Car and V637 Sct, and the overtone pulsator SU Cas fall on the blue edge of the instability strip. This is where pulsation calculations predict that overtone pulsation should be, but it is quite different from, for instance, the observed location of double mode pulsators found by Barrell 1981.

\section{Comparison of Variables and Nonvariables}

The same technique for determining Cepheid luminosities can also be used for nonvariable supergiants with hot companions. Recently we (Evans 1992c) have discussed the luminosities for two such systems, HD 183864 and HD 223047, also discussed by Ake 1988 and Parsons and Ake 1992. Figure 2 shows these results, the results from IUE luminosities for Cepheids, and the results for variables and nonvariables in clusters from Schmidt 1984. Both the IUE results and the Schmidt results have luminosities determined for variables and nonvariables using the same technique, and so the relation between the variables and the nonvariables should be accurate. As can be seen in Figure 2, Schmidt's conclusion that there is at most minimal overlap between Cepheids and nonvariables is still true. This does not, however, seem to be the case for the combined sample of Magellanic Cloud clusters (e.g. Mateo, et al. 1990). However, individual Magellanic Cloud clusters show a fairly clear separation between variables and nonvariables (Evans 1992c).

In Figure 2, the diagonal band in the HR diagram made up of both variable and nonvariable supergiants is what is expected from stars near the tips of the blue loops of the evolutionary tracks, as discussed by Schmidt. The hot edge of the blue loops is shown schematically by the solid line. (The solid point at $(0.2,-2.4)$ is ignored in this discussion as a discordant point.) This line is quite well defined observationally, and can serve as a useful constraint on evolutionary calculations. What do we know about the boundaries of the instability region? On the red side, there are both variables and nonvariables for a large range of luminosity which define the boundary of the instability strip (shown schematically by the dashed line). On the blue side, on the other hand, only for high luminosities are there nonvariables as well as variables (dotted line). For lower Cepheid luminosities, there are no nonvariables hotter than the instability region. In fact, it looks in Figure 2 as though the location of the hottest Cepheids is determined more by the edges of the blue loops than by the stability/instability boundary. Since the location of the blue loop supergiant region affects the shape of the instability strip, it may even explain the narrowing of the strip at low luminosities, as discussed above. (The narrowing of the instability strip is more prominent in the IUE and Feast and Walker compilation than the IUE and Schmidt compilation.) To confirm this suggestion, it is desirable to have luminosities for additional nonvariable supergiants particularly on the hot side of the instability strip if any exist. The blue loop locus, however, is only one possible influence on the 
shape of the instability region (see, for instance Morgan, this conference).

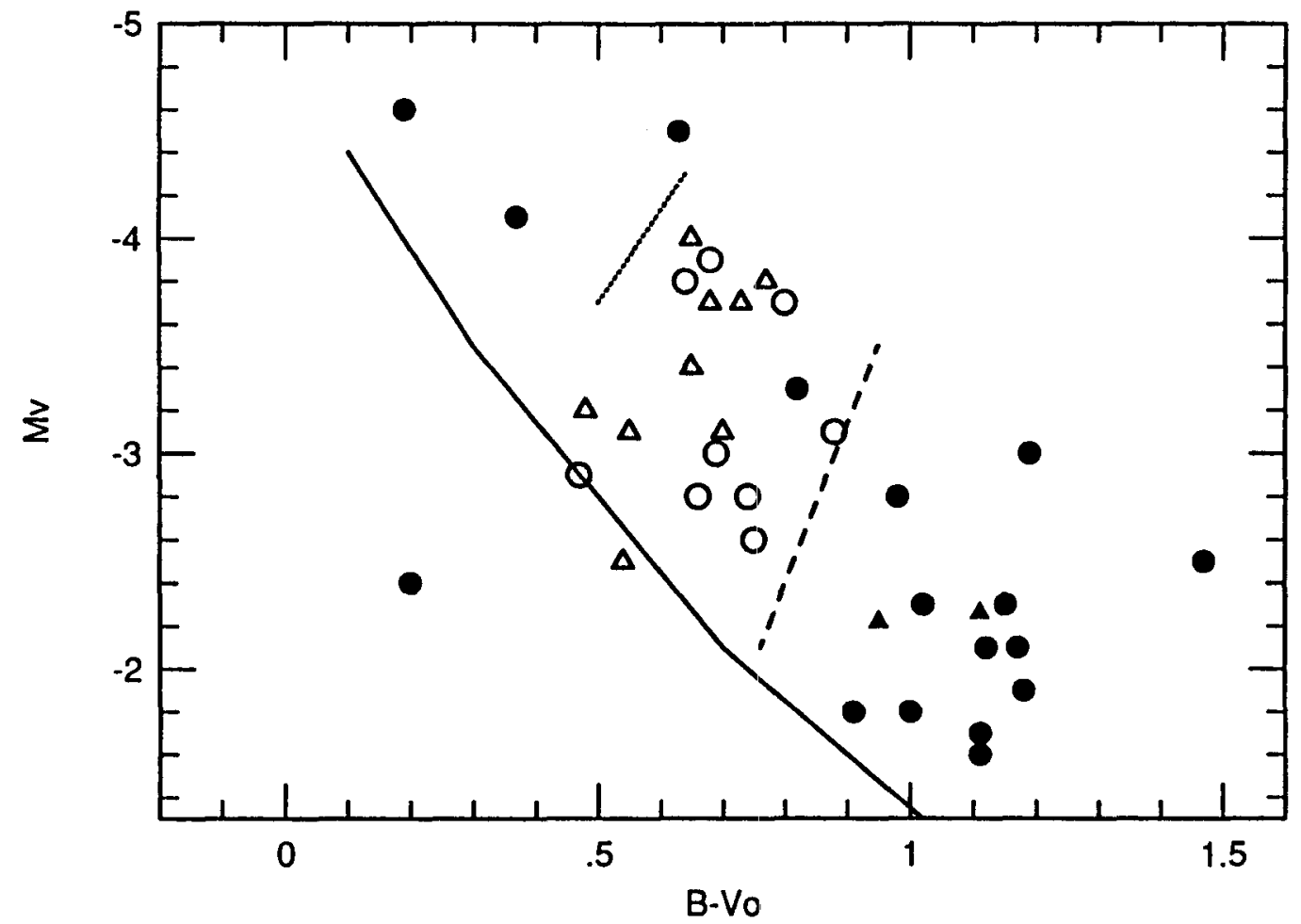

Figure 2. Comparison of Cepheids and nonvariable supergiants in the HR diagram. Triangles are from IUE observations, circles are from Schmidt; filled symbols are nonvariables, open symbols are variables. Lines show the schematic location of the tips of the blue loops (solid), the red edge of the instability strip (dashes) and the blue edge of the instability strip (dots) as determined from this sample.

\section{Cepheids with Evolved Companions}

A number of the Cepheid binary systems studied have hot companions which are evolved beyond the ZAMS. These have been treated differently than systems for which the large luminosity difference between the components confirms that the companions must be very close to the ZAMS. For systems with evolved companions, the luminosity of the Cepheid from the PLC has been used to place the components of the system 
on the HR diagram (Evans 1992d). These systems can then be compared
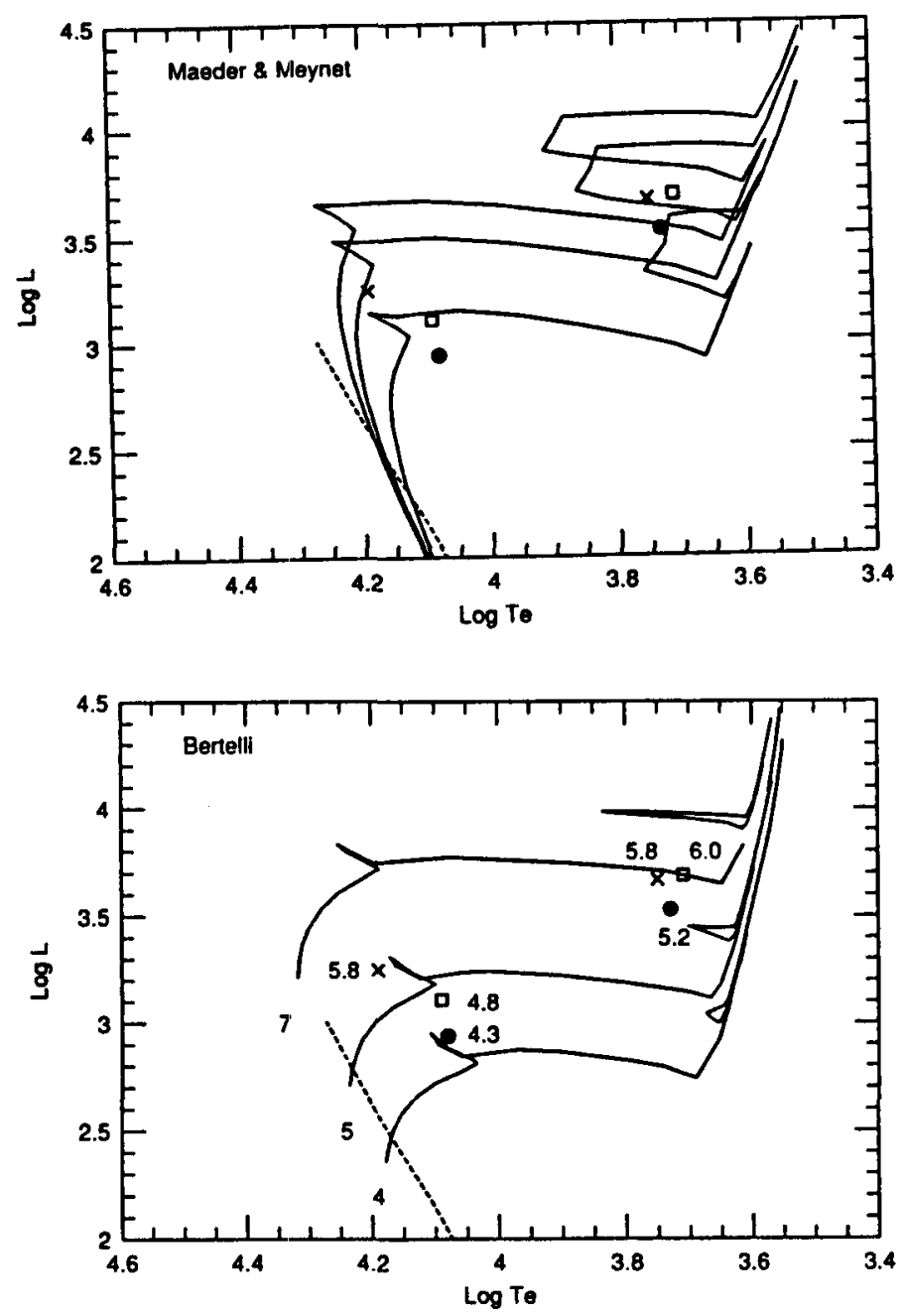

Figure 3 Theoretical IIR diagrams. The symbols represent the stars in the following systems: SY Nor: x's; RW Cam: open squares; SV Per: filled circles. a. The isochrones from Meader and Meynet are shown for log age 7.7, 7.8, and 8.0 years. b. The evolutionary tracks from Bertelli, et al 1986 are shown for masses 4, 5, and $\bar{\tau}$ solar masses. Near each star is the mass estimated from these tracks, again in solar masses.

with evolutionary tracks and isochrones. One of the parameters which is especially uncertain in evolutionary calculations is the amount of core convective overshoot for stars near the main sequence. One of the purposes of this study is to examine 
groups of binary systems containing Cepheids with similar periods. Since the mass and luminosity should be simple functions of the period, Cepheids within a small period range should have similar masses, and presumably should be well matched by evolutionary tracks with similar input physics.

Three short period systems BP Cir $(\mathrm{P}=2.4$ days $), A X$ Cir $(P=5.3$ days $)$, and V659 Cen ( $P=5.6$ days) agree well with isochrones with no convective overshoot, such as those of Stothers and Chin 1991. Because the luminosity difference between the Cepheids and companions is small for AX Cir and BP Cir, the companions must be near the end of their main sequence lifetimes, and the two stars in each system must have nearly the same mass. For V659 Cen, the luminosity difference is larger, and so, presumably, is the mass difference. As discussed by Evans, et al. 1992, for BP Cir this requires that the Cepheid be pulsating in the first overtone mode, making the fundamental mode 3.4 days. If a luminosity for the Cepheid is calculated assuming a period of 2.4 days, the companion is very close to the ZAMS, which disagrees with the small luminosity difference between the two stars. A period of 3.4 days makes both stars more luminous, and places the companion near the end of its main sequence lifetime. There are two other pieces of evidence that BP Cir is an overtone pulsator: the Fourier decomposition parameters (Antonello, et al. 1990), and the location of the Cepheid on the blue edge of the instability strip.

For the long period Cepheid SY Nor $(P=12.6)$, the two components fit an isochrone with a mild amount of overshoot such as that of Maeder and Meynet 1988 (Figure 3) better than one with no overshoot. This implies that the amount of convective overshoot may be a function of mass within the Cepheid mass range.

However, although these four systems can be understood within the framework of current evolutionary calculations, there are exceptions which are poorly fit by evolutionary tracks or isochrones. Figure 3 shows an example of the comparison of the SY Nor system with two systems with similar periods, RW Cam ( $\mathrm{P}=16.4$ days) and $S V$ Per $(P=11.1$ days $)$. Figures $3 a$ and b respectively show isochrones based on the calculations of Maeder and Meynet 1988 with mild overshoot and some mass loss, and evolutionary tracks of Bertelli, et al., 1986 with a large amount of overshoot. Note that recalculation of the Maeder-Meynet tracks (Meynet 1992) with new input physics, including the new opacities of Iglesias and Rogers 1991, removes the luminosity difference between the second and third crossing of the instability strip. That is, both branches of the blue loop lie very close to the second crossing in Figure 3b. Since the luminosities of the Cepheids in the SY Nor, RW Cam, and SV Per systems are all similar, the companions should fit a similar isochrone. Clearly in Figure 3a the companions RW Cam B and SV Per B are much too cool for the isochrone fitting the Cepheids. The RW Cam B and SV Per B temperatures are determined from well exposed IUE spectra, and the temperatures are in the temperature range where the IUE spectra are very sensitive to temperature, so the temperatures are well determined. (The SY Nor spectrum is not as well exposed.) A wide main sequence band is often taken as the signature of a large amount of convective overshoot. For this reason, we have also compared the same data with 
the Bertelli, et al. tracks, to see whether tracks with a large amount of overshoot (and calculations from a different code) result in better agreement. Figure $3 \mathrm{~b}$ shows that the main sequence band is wide enough to include the companions for RW Cam and SV Per. However, the luminosity differences are not consistent with the tracks. The masses of each star estimated from the tracks are included in Figure 3b. The poor agreement with the SY Nor system is reflected in the fact that the stars have no mass difference at all with these tracks. For RW Cam and SV Per, on the other hand, the two stars differ by approximately a solar mass, which is too large for a blue loop star and a star at the end of its main sequence lifetime. The evolved nature of RW Cam B and SV Per B is confirmed by the fact that the IUE flux distributions match luminosity class III stars better than class $V$ stars.

Two other systems may show the same inconsistency with evolutionary tracks, KN Cen $(P=34.0$ days $)$ and AW Per $(P=6.5$ days $)$. In both cases there are possible alternate explanations, which are, however, not entirely successful. Because KN Cen is the longest period star in the sample, any parameter such as overshoot which is a function of mass may have an extreme value in its case. The companion to AW Per may itself be a binary. While this would give the companion increased luminosity for its color, even the maximum correction for this effect would not result in a companion on the main sequence. In summary, two and possibly four of the eight systems with evolved companions studied are not well matched with current evolutionary tracks. Furthermore, the discrepant cases are spread out throughout the range of period, so a simple variation of some parameter as a function of mass is not a likely cause. Changes in evolutionary tracks because of rotation is an example of a possible cause.

\section{Summary}

Studies of the hot companions of Cepheids from IUE spectra have been used to determine the luminosities of Cepheids. Included in the sample are an overtone pulsator and a double mode pulsator, which are found on the blue edge of the instability strip in the HR diagram. A comparison between the location of variables and nonvariables (from the IUE studies and the cluster studies of Schmidt) shows very little overlap. Binary systems containing a Cepheid and a companion within the main sequence band but evolved beyond the ZAMS cannot be matched with current isochrones in all cases.

\section{References:}

Ake, T. 1988, in "New Directions in Spectrophotometry" eds. A. G. D. Philip, D. S. Hayes, and S. J. Adelman, (Schnectady: L. Davis Press), p. 27.

Antonello, E., Poretti, E. and Reduzzi, L. 1990, Astr. Ap., 236, 138.

Barrell, S. L. 1981, M. N. R. A. S., 196, 357.

Bertelli, G., Bressan, A., Chiosi, C., and Angerer, K. 1986, Astron. Ap. Suppl., 66, 191.

Böhm-Vitense, E. 1985, Astrophys. J., 296, 169.

Böhm-Vitense, E. and Proffitt, C. 1985, Astrophys. J., 296, 175.

Evans, N. R. 1991, Astrophys. J., 372, 597. 
-. 1992a, Astrophys. J., $389,657$.

-. 1992b, Astrophys. J., $385,680$.

-. 1992c, preprint

-. 1992d, preprint

Evans, N. R., Arellano Ferro, A., and Udalska, 1992, Astron. J., 103, 1638.

Feast, M. W. and Walker, A. R. 1987, Ann. Rev. Astr. Ap, 25, 345.

Fernie, J. D. 1990, Astrophys. J., 354, 295.

Iglesias, C. A. and Rogers, F. J. 1991, Astrophys. J., 371, 408.

Maeder, A. and Meynet, G. 1988, Astron. Ap. Suppl., 76, 411.

Mateo, M., Olszewski, E. W., and Madore, B. F. 1990, in A. S. P Conf. Ser., 11, 214.

Meynet, G. 1992, private communication.

Parsons, S. B. and Ake, T. 1992, Bull. A. A. S. 24, 769.

Schmidt, E. G. 1984, Astrophys. J., 287, 261.

Stothers, R. B. and Chin, C. 1991, Astrophys. J., 381, L67.

Question:

Turner: I have a comment and a question. First, one paraneter which evolutionary models do not include is rotation, which can displace a star to cooler effective temperatures in the HR diagram. Also a B dwarf which is rapidly rotating and seen equator-on appears spectroscopically like a giant. This may help you explain the apparent anomalies you find for some of the Cepheid companions. Second, you and I disagree about the pulsation mode of SU Cas, and this seems to be tied to differences in the magnitude difference between the Cepheid and its companion. I am puzzled by the large $\Delta V$ you find for SU Cas, since it seems to contradict your $\mathrm{K}$-line spectroscopic observations which implied a smaller $\Delta \mathrm{V}$.

Evans: Yes, rotation might explain the systems which fit evolutionary tracks poorly. For SU Cas, the magnitude difference between the two stars is much better determined from the IUE spectra than from the Ca II K line observations. For the $\mathrm{K}$ line spectra, the companion is only prominent for a small wavelength region in the line core, and any quantitative measure must take into account grating scattered light as well as the shape of the Cepheid line cores. 